\title{
ANALYSING SUPPLY CHAIN PHASES FOR DESIGN OF EFFECTIVE AUTONOMOUS SHIP TECHNOLOGY IN NEW TRANSPORT SYSTEM SOLUTIONS
}

\author{
Lars Andreas Lien Wennersberg \\ SINTEF Ocean \\ Trondheim, Norway \\ lars.andreas.wennersberg@sintef.no
}

\author{
Håvard Nordahl \\ SINTEF Ocean \\ Trondheim, Norway \\ haavard.nordahl@sintef.no
}

\author{
Ørnulf Jan Rødseth \\ SINTEF Ocean \\ Trondheim, Norway \\ ornulfjan.rodseth@sintef.no
}

\author{
Victor Bolbot \\ Maritime Safety Research Centre \\ University of Strathclyde \\ Glasgow, Scotland \\ victor.bolbot@strath.ac.uk
}

\author{
Gerasimos Theotokatos \\ Maritime Safety Research Centre \\ University of Strathclyde \\ Glasgow, Scotland \\ gerasimos.theotokatos@strath.ac.uk
}

\begin{abstract}
Maritime autonomous surface ships provide new capabilities for transport systems design, and by that the potential to dramatically change ship logistics. Advantages of autonomous ships include operational flexibility with more and smaller ships, combined with new ship hull and superstructure designs, allowing more cargo to be transported at lower cost and reduced emissions. It is also easier to operate smaller ships with alternative energy sources such as batteries, and by that further reducing the emissions. However, the investment cost of an autonomous ship system is perceived to be higher compared with conventional ships. Combined with limited operational experience, this creates a challenging threshold for launching new projects.

The purpose of this work is to lower this threshold by providing a better understanding for the analysis tools employed in new transport systems design. Structured analysis of the supply chain phases and tasks enables the involved stakeholders to evaluate the cost and benefits associated with the increased automation in a specific phase of the transport system. This will additionally form a basis for developing business models and priorities for gradual introduction of key enabling technologies through retrofitting of conventional ships or designing and build-
\end{abstract}

ing new smart ships.

A scalable method for the systematic supply chain analysis of waterborne transport systems is introduced. The method is developed through a mapping and analysis of one inland waterways use-case and one short sea shipping use-case, and used as a basis for the systematic identification of all the supply chain phases and all tasks carried out in each phase of the transport system. A design process for autonomous ship systems consisting of the supply chain analysis, the task distribution and the cost benefit analysis is also presented. The effectiveness of this process in the design and incorporation of autonomous ship technologies in new transport systems solutions is demonstrated.

\section{INTRODUCTION}

The European Union (EU) has set an ambitious target; to move fifty percent of the long distance goods transported on roads to other transportation means by 2050 [1]. Modal shifts from road to short sea shipping (SSS) and Inland Waterways (IWW) transport is essential to achieve this goal. EU statistics do however reveal that progress is slow [2]. SSS and IWW transport needs to gain competitiveness against road transport to speed up progress, while at the same time adapting to the new Inter- 
national Maritime Organisation (IMO) target for reducing green house gas emissions by fifty percent till 2050, compared with 2008.

A recent study indicates that the profitability and environmental profile of the SSS transport can be improved by combining slender ship designs and autonomous ship systems, thus resulting in increased competitiveness against road transport [3]. Despite the potential to increase the efficiency of transport systems and reduce emissions, the growing interest in maritime autonomous surface ships (MASS) has so far resulted in few commercial projects.

As of today, limited operational experience with the key enabling technologies for autonomous ship systems is available [4], whereas the cost for installing these systems is expected to be higher in comparison with the respective conventional ships. This combined is perceived as an investment risk and it creates a challenging threshold for launching new projects that could contribute positively to EU and IMO targets.

The purpose of this work is to lower this threshold by providing a better understanding for the analysis tools employed in new transport systems design. The success of any transport system relies on cost-effective and safe system designs. To achieve this for MASS, we need to answer two fundamental design questions:

1. How shall tasks be distributed between ship and shore?

2. How shall tasks be distributed between system automation and humans?

The objective of this work is to provide the grounds to answer these design questions using a scalable and systematic method for analysis and breakdown of supply chain phases and tasks in waterborne transport systems.

\subsection{Supply Chains}

A supply chain is made up by flows of information and material, and is designed to fulfill one or several objectives [5]. According to [6], "Unmanned ships have an immense potential as a new component in completely new transports systems with much higher integration into logistics chains.”. A supply process consisting of a Production Planning and Inventory Control process connected to a Distribution and Logistics process, where the former describes how goods is produced and the latter how goods is transported from a producer to a consumer, i.e. the process of transporting goods from one location to another, is presented in [7].

To understand the scope of integration in the supply chain, we need knowledge on the intended purpose of the MASS, and specifically its role as a transport system component in the supply chain. This knowledge can be gained through supply chain analysis. A waterborne transport model that captures the role of the MASS in a supply chain will have to consider both the flow of goods and the flow of information required to transport the goods from one location to another. During MASS design, it is therefore essential to systematically identify all tasks that must be carried out, and under what conditions these tasks are to be performed to meet the performance objectives of the supply chain. This could be done both for existing transport systems and for development of new transport systems.

\subsection{Maritime Autonomous Surface Ships}

Maritime Autonomous Surface Ships (MASS) are new ship types that can be used in the design of transport systems fulfilling the needs of a supply chain. The very near future will bring two new ship types in the MASS category on the market; Smart ships and unmanned ships. Both ship types will in varying degree utilize autonomous ship technology. Compared to conventional ships, a smart ship will have more on-board automation and decision support systems to support the crew in their daily tasks. This will on short term lead to longer periods and more complex operations with an unattended bridge and machinery room. On longer term it will lead to crew reductions, paving the way towards unmanned ships. Unmanned ships will be designed specifically for a dedicated transport system. They will require support from remote control centers and possibly more automated shore infrastructure compared to smart ships.

Unmanned ships and ships with a significant crew reduction compared to conventional ships has the advantage of enabling higher operational flexibility by making smaller ships competitive with larger ships [3]. In a transport system several small ships can be used to achieve higher utilisation of the ship capacity and higher frequency. Another key advantage is that the hull can be re-designed to increase the ships relative cargo capacity, which reduces both the transportation cost and emission per transported unit [3]. It is also easier to introduce alternative energy sources such as batteries on smaller ships [6], and by that reducing emissions further.

Technology and humans constitute the system components in this context, and they carry out system operations by means of automation in the case of technology and procedures in the case of humans. Today, it is not clear what the costs and benefits of determining the appropriate level of system automation are. Neither is it clear what the costs and benefits of distributing the tasks between ship and shore are, and thus we lack knowledge to make proper investment decisions.

Two studies have compared the costs of running autonomous bulk ships with the costs of running conventional bulk ships [8], [9]. Another approach would be to introduce higher levels of autonomy in specific phases of a transport system based on a cost-benefit analysis of task breakdown.

The advantage of this approach is that structured analysis of supply chain phases and tasks enables stakeholders to evaluate the cost and benefits associated with increased automation and 
task distribution between ship and shore. This in turn forms a basis for development of business models that could set priorities for gradual introduction of key enabling technologies (KET) through retrofitting of conventional ships or new building of smart ships. Gradual introduction of KETs would furthermore speed up operational experience and at the same time facilitate quantification of early design cost and benefit estimates, and thus lower the threshold to launch new MASS projects as the knowledge base for investment decisions improves.

\section{DESIGN METHOD FOR MASS SYSTEMS}

The method proposed in this paper for early stage design and cost-benefit analysis of autonomous ship systems is illustrated in Fig. 1. The process consists of three main steps:

1. Supply Chain Analysis

2. Task Distribution

3. Cost Benefit Analysis

Several design parameters can be adjusted to achieve the desired functionality of an autonomous ship system. Among those are distribution of tasks between ship and shore, and between system automation and humans. Designs can also be simplified by reducing the operational complexity, such as the weather windows that the autonomous ship system must handle. The costs of implementing a design could be too high compared to the number of days a ship would have to deal with the complexity, and it could be beneficial to choose a simpler design. This would in effect mean a trade-off with operational availability of the ship.

The possibility to adjust design factors and limit operational complexity provides design flexibility. This flexibility must be systematically managed during design, and be supported by proper definitions for task and responsibility distribution.

\subsection{Man and Machine Responsibility}

Autonomy levels describe the degree of automation in a system, and can be used to ensure a correct understanding of the division of responsibility between man and machine.

In the automotive industry the SAE suggests a taxonomy that fits well with our needs [10]. This is further adapted to the maritime domain in [11], where autonomy levels for MASS are suggested. In [10] the Operational Design Domain (ODD) is defined as

Operating conditions under which a given driving automation system or feature thereof is specifically designed to function, including, but not limited to, environmental, geographical, and time-of-day restrictions, and/or the requisite presence or absence of certain traffic or roadway characteristics.
Which can be adapted to the maritime domain by swapping driving with navigating and roadway with fairway.

The term Operational Design Domain is proposed changed to Operational Envelope (OE) as part of ongoing work in ISO [12] on MASS terminology. Operational Envelope is thus used in the remained of this paper.

While the ODD definition in [10] will be the basis for our understanding of the OE, we will adapt the concepts: Autonomy Levels, Dynamic Navigation Task (DNT) and DNT fallback from [11], where the DNT are all the individual tasks that needs to be performed to handle the complexity of the operational envelope. This can furthermore be split into the tasks that is to be handled by crew, Operator Exclusice DNT (OE-DNT), or by control and automation systems, Control System DNT (CS-DNT) [11].

\subsection{Design Flexiblity}

The flexibility that MASS systems offers must be systematically managed during the design phase. The operational viewpoint of the SEATONOMY methodology [13] is of particular interest in early phase concept studies and design of MASS. The viewpoint aims to define the operation design and requirements through breakdown of operations using Autonomous Job Analysis [13] supported by the Canvas method [14], and map humanmachine collaboration to autonomy levels. This work will build upon the operational viewpoint in the SEATONOMY methodology.

\subsection{Risk Impact}

MASS can be viewed as cyber-physical systems of systems. Hazard identification and risk analysis are identified as important safety assurance methods during the design phase of cyberphysical systems [15]. Risk based design was applied to an unmanned ship in [16]. The need for systematic identification of hazards in the early design process of autonomous ships is highlighted in [17], and a process for this is proposed where amongst other mitigating actions are linked to cost.

Guidance on how to treat hazards during a design process is given in [18]. If the distribution of tasks for a MASS system gives an unnacceptable risk, then an immediate correction, i.e task redistribution, is required to reduce the risk to an acceptable level. If a risk is accepted, the risk will have an impact on design or operational cost, as it must be managed to ensure safety. As an example of the latter, it is possible that the ship in operation may end up in a situation outside of the operational envelope. This means that the ship is operating outside design conditions. In this situation, a DNT fallback must be activated, either by crew or the automation system, to bring the ship to a safe state. The DNT fallback may have a cost both during design and use in the operation. 


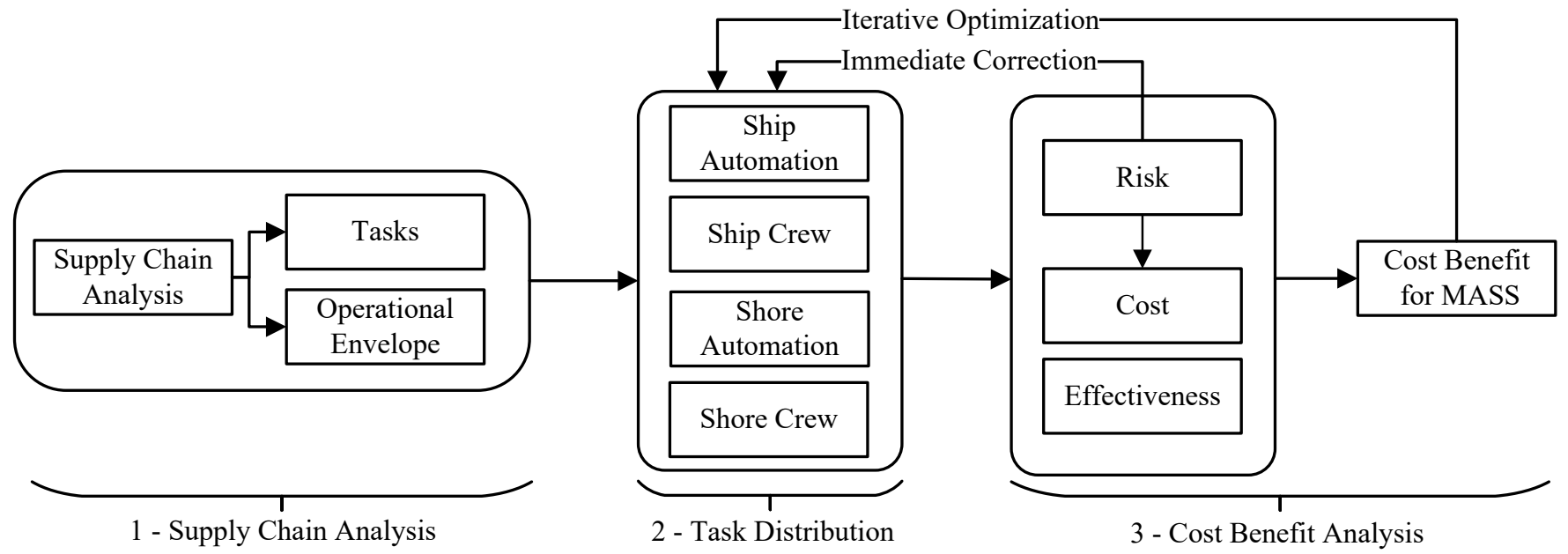

FIGURE 1: Early stage design and cost-benefit process for autonomous ship systems.

TABLE 1: Phases of the waterborne transport model.

\begin{tabular}{|l|l|}
\hline Phase 0 & Logistics Management \\
\hline Phase 1 & Carry out activities at location \\
\hline Phase 2 & Depart from location when activities are completed \\
\hline Phase 3 & Sail towards next location \\
\hline Phase 4 & Approach location \\
\hline Phase 5 & Carry out maintenance in parallel with phases 1-4. \\
\hline
\end{tabular}

\subsection{Waterborne Transport Model}

Based on a supply chain analysis of one IWW use-case and one SSS use-case, a Waterborne Transport Model was developed in [19] to capture the role of a ship as a transport system component in a supply chain. The waterborne transport model can be broken down into five distinct ship operation phases and one supply chain management phase. The phases are listed in Table 1 and the transitions between the phases is illustrated in Fig. 2 The generic term Location is used to describe where the ship is, such as in harbour, at quay, in water-lock and so forth.

The supply chain management phase goes in parallel with the ship operation phases. These are organisational processes which includes logistics and planning of crew manning, maintenance and so forth. The supply chain management phase is tightly connected to all five ship operation phases, especially with respect to flow of information between ship and office.

\subsection{Description of Design Process}

The merger of supply chain analysis and the design process for MASS and autonomous ship systems is illustrated in Fig. 1.

The first step in the process, which is the main topic of this paper, is the supply chain analysis. The objective of the supply chain analysis is to identify the tasks that needs to be carried out by the ship, and to define the operational envelope, i.e the operational complexity that the ship needs to handle. The output of the supply chain analysis is the input to the task distribution process.

The second step is to assign the identified tasks to ship automation, ship crew, shore automation and shore crew. The task assignment should cover all tasks and operational envelopes identified in the supply chain analysis. The new task assignment is input to the cost-benefit analysis.

The third step is to perform a cost-benefit analysis of the new task distribution. The task distribution constitutes the design, and the design cost must be evaluated towards the design effectiveness. The cost-benefit analysis also contains a risk analysis step. If the design results in too high financial risks, inadequate operational up-time or unacceptable safety levels, then immediate correction is needed and the process must return to step two. If the design results in an acceptable risk level, the cost of managing the identified risks should be quantified and included in the cost estimate.

The result of the cost benefit analysis might be nonsatisfactory. In such cases iterative optimization over step two and step three is needed until a final conclusion on the design viability can be made. A viable design should be able to provide a definite answer to the design questions:

1. How shall tasks be distributed between ship and shore?

2. How shall tasks be distributed between system automation and humans?

The remainder of this paper will go in depth on the supply chain analysis process and illustrate the process with results from an use-case. 


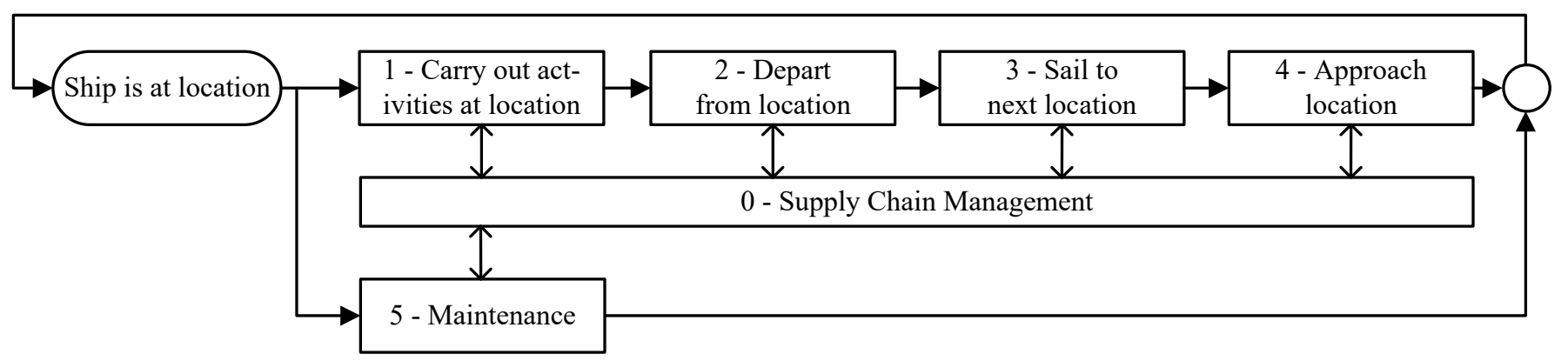

FIGURE 2: Transitions between phases in the waterborne transport model.

\section{SUPPLY CHAIN ANALYSIS}

The proposed method for analysing the supply chain for waterborne transport systems is based on organising the transport system as shown in Fig. 2. It is essential to identify all flows to, from and within the waterborne transport model to understand which tasks needs to be carried out and how this will affect the overall performance of the supply chain. Each of the phases are split into specific tasks, and each task further into subtasks until an adequate level of details is achieved. This could either be navigational tasks, shore-to-ship cargo handling tasks or any other task related to the transport system.

\subsection{Step 1 - Gather and Organize Information}

The first step is to gather all necessary information about the existing transport system or establish requirements for a new transport system. To do this, a survey with the following nonexhaustive topics should be carried out [19]:

1. Goods

2. Pick-up and drop off locations

3. Factories and customers

4. Ships

5. Supply chain and distribution systems

6. Market profile and economy

7. Performance indicators

8. Regulatory and contractual considerations

The output of the survey is a mapping of transport system operations, interactions and objectives. This step is a concretization of how to establish the context definition of the operational viewpoint in [13] for MASS and autonomous ship systems.

The information should be organized according to the phases of the waterborne transport model. An example of information relevant for Phase 1: Activities at location in the waterborne transport model is listed in Table 2 .

\subsection{Step 2 - Identify Tasks}

The second step is task identification. This can start once the supply chain is analysed and the information is organized
TABLE 2: Structure of mapped information for phase 1 in the waterborne transport model.

\begin{tabular}{|c|l|}
\hline Information category & Details \\
\hline Loading of goods & $\begin{array}{l}\text { Type of goods } \\
\text { Amount of goods } \\
\text { Effective loading rate }\end{array}$ \\
\hline Offloading of goods & $\begin{array}{l}\text { Type of goods } \\
\text { Amount of goods } \\
\text { Effective offloading rate }\end{array}$ \\
\hline Pass obstacle & $\begin{array}{l}\text { Type of obstacle to pass } \\
\text { (e.g. bridge, water-lock, other ship) }\end{array}$ \\
\hline Bunkering & $\begin{array}{l}\text { Amount to bunker } \\
\text { Effective bunkering rate }\end{array}$ \\
\hline Service and Repair & Time required \\
\hline Crew Change & Time required \\
\hline Wait & $\begin{array}{l}\text { Time until activities can commence } \\
\text { or ship is ready to depart }\end{array}$ \\
\hline
\end{tabular}

according to the waterborne transport model. Identification of the tasks, or the DNT, can be simplified by applying the method presented in [19]. The output of this step is a structured table of all tasks that must be performed to navigate and handle goods safely while fulfilling the overall purpose of the transport system and supply chain.

The information gathered for each phase in the waterborne transport model are split into specific tasks, and each task further into subtasks until an adequate level of details is achieved. This enables a systematic description of the transport system down to a level which corresponds to all the individual work tasks that must be performed either by crew or automation, and to enable 
TABLE 3: Operational Envelope Categories

\begin{tabular}{|c|c|}
\hline OE Category & OE Complexity \\
\hline \multirow[t]{3}{*}{ Weather } & Wind $[\mathrm{m} / \mathrm{s}]$ \\
\hline & Wave $[\mathrm{m}]$ \\
\hline & Current $[\mathrm{m} / \mathrm{s}]$ \\
\hline \multirow[t]{3}{*}{ Traffic } & Ships \\
\hline & Leisure \\
\hline & Life \\
\hline \multirow[t]{6}{*}{ Geographic } & Open sea \\
\hline & Sheltered \\
\hline & Close to asset \\
\hline & Reefs/Shallow \\
\hline & Narrow \\
\hline & High obstruction \\
\hline \multirow[t]{2}{*}{ Operating hours } & Day time \\
\hline & Nightlay \\
\hline \multirow[t]{2}{*}{ People onboard } & Passengers \\
\hline & Crew \\
\hline
\end{tabular}

evaluation of autonomy level for each of these subtasks. The subtasks, i.e. the DNT, must be handled either as OE-DNT or CS-DNT.

\subsection{Step 3 - Define Operational Envelopes}

The third step is definition of operational envelopes. Note that this step can and often will be done in parallel with task identification. An operational envelope describes what the ship needs to handle in terms of operational complexity. Definition of operational envelopes can start once the supply chain is analysed and the information is organized according to the waterborne transport model. A non-exhausting list of operational envelope categories is given in Table 3 as a suggested approach to describe the operational complexity.

\subsection{Step 4 - Link Tasks and Operational Envelopes}

The fourth and final step is to link tasks with operational envelopes. The categories of the operational envelope can be linked to one or several phases in the supply chain. Weather conditions might be different for loading and unloading operations. Several tasks might be carried out under the same operational complexity, and can thus be linked to the same operational envelope. It could also be that subtasks of a task will be subject to different operational envelopes.

This step is essential to understand the complete picture and to provide the ground for the next step in the design process which is task distribution.

\section{RESULTS AND DISCUSSION}

Two use-cases have been considered as part of the development of the waterborne transport model and the systematic supply chain analysis method; one short sea shipping use case where bulk goods is transported along the Norwegian coast and one inland waterways use case where palletized goods and containers are transported on rivers and canals in the Flemmish region. Both ships are to be rebuilt to demonstrate autonomous sailing, and the purpose of the analysis was to understand the ship operations asis-today to be able to further develop the transport system using autonomous ship technology. This section will illustrate some of the results obtained from the supply chain analysis of the IWW use-case [19].

\subsection{IWW Task Identification}

The supply chain analysis of the inland waterways use-case resulted in the identification of the phases and tasks as shown in Table 4. The tasks were further analysed and structured into subtasks. A sample set of how the navigate task and pass water-lock task was divided into subtasks is given in Table 5 The autonomy level (AL) for each subtask was also evaluated, with the intention to use this as a basis for evaluating the economical benefits of increasing the autonomy level. This ship is operated by a crew of one, and most tasks are carried out manually with support from automation systems. This is also reflected as AL 0 and 1 in Table 5 according to the definitions in [11].

It is worth noticing that the ship is dependent on shore infrastructure and shore personnel when passing through the waterlock. This will be of particular importance during the design phase and cost-benefit evaluation, as increased automation and improved and possible automatic communication towards shore must be taken into account. A full breakdown of all subtasks are given in [19].

\subsection{IWW Operational Envelope}

Waterborne transport on inland waterways are subject to several external and internal factors that adds up to the operational complexity. Table 6 illustrates a set of operational envelope categories for the use-case.

Notice that the Navigate task is linked to two different weather categories. The main reason for this is that there normally is no current and almost constant water depth in canals, whereas current speeds can reach up to $7.0 \mathrm{~m} / \mathrm{s}$ and the water depth will vary in rivers. The complexity of navigating on rivers 
TABLE 4: Identified phases and tasks for inland waterways usecase analysis

\begin{tabular}{|c|c|c|c|}
\hline Phase & Description & Task & Description \\
\hline 0 & Logistics & 0.1 & Logistics management \\
\hline 1 & Loading & $\begin{array}{l}1.1 \\
1.2 \\
1.3 \\
1.4\end{array}$ & $\begin{array}{l}\text { Rolling containers } \\
\text { Big Bags } \\
\text { Pallets } \\
\text { Other }\end{array}$ \\
\hline 1 & Offloading & $\begin{array}{l}1.5 \\
1.6 \\
1.7 \\
1.8\end{array}$ & $\begin{array}{l}\text { Rolling containers } \\
\text { Big Bags } \\
\text { Pallets } \\
\text { Other }\end{array}$ \\
\hline 1 & Bunkering & $\begin{array}{c}1.9 \\
1.10\end{array}$ & $\begin{array}{l}\text { Order bunker service } \\
\text { Bunkering process }\end{array}$ \\
\hline 2 & Departure & 2.1 & Departure from quay \\
\hline 3 & Sailing & $\begin{array}{l}3.1 \\
3.2 \\
3.3\end{array}$ & $\begin{array}{l}\text { Navigate } \\
\text { Pass water-lock } \\
\text { Pass under bridge }\end{array}$ \\
\hline 4 & Approach & 4.1 & Approach to quay \\
\hline 5 & $\begin{array}{l}\text { Maintenance and } \\
\text { service }\end{array}$ & $\begin{array}{l}5.1 \\
5.2 \\
5.3\end{array}$ & $\begin{array}{l}\text { Daily maintenance } \\
\text { Service and repair } \\
\text { Dry docking }\end{array}$ \\
\hline
\end{tabular}

and canals differs. The wind speed and wave height is marked as $\mathrm{NN}$ as exact numbers have not been established. Notice also that passing a water lock can lead to small current speeds in canals as the water lock is filled up and emptied.

Several categories also applies to all tasks. This means that irrespective of which tasks that is carried out, this complexity will remain constant throughout all phases of the operation as it is analysed.

\subsection{Discussion}

The supply chain analysis method serves to provide an overview of what is to be done under which circumstances. The breakdown structure can be used to analyse parts of ship systems or parts of a transport system, but can also be expanded to complete the whole transport system. The method allows for analysis and identification of navigational tasks, communication tasks, cargo handling tasks and other relevant tasks. The analysis
TABLE 5: Identified subtasks with corresponding autonomy levels (AL) for Task 3.1 and 3.2. Based on [19].

\begin{tabular}{|c|c|c|c|c|}
\hline Task & Description & Subtask & Description & $\mathrm{AL}$ \\
\hline \multirow[t]{6}{*}{3.1} & Navigate & 3.1 .1 & Select/update route & 0 \\
\hline & & 3.1 .2 & Follow route & 1 \\
\hline & & 3.1 .3 & Situational awareness & 0 \\
\hline & & 3.1 .4 & Avoid obstacles & 0 \\
\hline & & 3.1 .5 & Communicate & 0 \\
\hline & & & with others & 0 \\
\hline \multirow[t]{12}{*}{3.2} & Pass & 3.2 .1 & Notify lock operator & 0 \\
\hline & water- & & 30 min. in advance & \\
\hline & lock & 3.2 .2 & Notify at arrival & 0 \\
\hline & & 3.2 .3 & Check if lock is ready & 0 \\
\hline & & 3.2 .4 & Wait & 0 \\
\hline & & 3.2 .5 & Operator opens lock & 1 \\
\hline & & 3.2 .6 & Ship enters lock & 1 \\
\hline & & 3.2 .7 & Operator closes lock & 1 \\
\hline & & 3.2 .8 & Water level is adjusted & 1 \\
\hline & & 3.2 .9 & Operator opens lock & 1 \\
\hline & & 3.2 .10 & Ship exits lock & 1 \\
\hline & & 3.2 .11 & Operator closes lock & 1 \\
\hline
\end{tabular}

method is thus scalable.

The results of the IWW use-case shows the importance of linking task execution with operational envelopes to obtain full understanding of the operational context. The supply chain objectives and flows may impose constrains to the MASS design process. This is also true for the identified tasks and operational envelopes, but it doesn't imply that the context can't change as part of the design process. Distributing tasks between shore, ship, crew and automation can inherently also imply modification of the operational envelope. A crew of one operated the ship between hours 0600 and 2200 in the use-case example. It does not mean that it needs to remain the same in the next step of the MASS design process which is task distribution. It could be that the operational hours can be adjusted and that the onboard crew could be adjusted to zero, whereas a new operational envelope category "People onshore" will have to be introduced.

The design process in Fig. 1 illustrates task distribution between ship, shore, human and system automation. The step from supply chain analysis to task distribution can be considered as 
TABLE 6: Identified operational envelopes in the IWW use-case.

\begin{tabular}{|c|l|c|l|c|}
\hline Task & Desc. & OE Category & OE Complexity & Value \\
\hline 3.1 & Navi- & Weather & Wind $[\mathrm{m} / \mathrm{s}]$ & NN \\
& gate & on canals & Wave $[\mathrm{m}]$ & $\mathrm{NN}$ \\
& & & Current $[\mathrm{m} / \mathrm{s}]$ & 7,0 \\
\hline \multirow{2}{*}{3.1} & Navi- & Weather & Wind $[\mathrm{m} / \mathrm{s}]$ & NN \\
& gate & on rivers & Wave $[\mathrm{m}]$ & NN \\
& & & Current $[\mathrm{m} / \mathrm{s}]$ & 0,0 \\
\hline 3.2 & Pass & Weather & Wind $[\mathrm{m} / \mathrm{s}]$ & NN \\
& water- & & Wave $[\mathrm{m}]$ & 0 \\
& lock & & Current $[\mathrm{m} / \mathrm{s}]$ & 0,5 \\
\hline \multirow{2}{*}{ All } & All & Geographic & Sheltered & Yes \\
& & & Close to asset & Yes \\
& & & Reefs/Shallow & Yes \\
& & & Narrow & Yes \\
& & & High obstruction & Yes \\
\hline \multirow{2}{*}{ All } & \multirow{2}{*}{ All } & Operating & Day time & $06-22$ \\
& & hours & Nightlay & $22-06$ \\
\hline \multirow{2}{*}{ All } & \multirow{2}{*}{ All } & People & Crew & 1 \\
& & onboard & & \\
\hline
\end{tabular}

incremental, whereas the process of task distribution and costbenefit analysis is iterative. It could however be necessary to return to the supply chain analysis step if the initial analysis is insufficient, or if the context, as discussed above, change as part of the design process.

\section{CONCLUSION}

This paper has introduced a scalable method for systematic supply chain analysis of waterborne transport systems. A design process for autonomous ship systems consisting of the supply chain analysis, the task distribution and the cost benefit analysis has been also presented.

The first main finding of this paper is a method that structures ship operation into phases, and enables

- identification of tasks, subtasks and autonomy levels belonging to each phase, and

- identification of the operational envelopes and their applicability to tasks and subtasks.
The second is that the result of the supply chain analysis provides the basis we need to be able to start on task distribution between ship and shore, and human and automation.

The third is that understanding the supply chain, i.e what needs to be done under what circumstances, is essential to progress with the question on how task distribution shall be done to produce a cost-effective and safe design of autonomous ship systems, and key to enable design of effective autonomous ship technology in new transport system solutions.

Whilst the main principles behind the new design process have been provided, further extensions and refinements are to be made and tested on the two use-cases. Future work needs to consider how to implement the task distribution and cost-benefit analysis processes given the basic principles provided in this paper. Further research is also required to consider the impact of fleet size and the required changes in the infrastructure on ship logistics and design

\section{ACKNOWLEDGMENT}

The work presented in this article is part of the AUTOSHIP project funded by EU Horizon 2020 with grant number 815012 . Thanks to Blue Line Logistics NV for providing necessary usecase information.

\section{REFERENCES}

[1] European Commission, 2016. Commission staff working document refit ex-post evaluation of combined transport directive 92/106/eec final report.

[2] European Commission, 2019. Statistical pocketbook 2019.

[3] Gribkovskaia, V., Borgen, H., Holte, E. A., Lindstad, E., and Nordahl, H., 2019. "Autonomous ships for coastal and short-sea shipping". In The Society of Naval Architects and Marine Engineers., no. SNAME-SMC-2019-068.

[4] Heffner, K., and Rødseth, Ø. J., 2019. "Enabling technologies for maritime autonomous surface ships". Journal of Physics: Conference Series, 1357, oct, p. 012021.

[5] Cerchione, R., and Esposito, E., 2016. "A systematic review of supply chain knowledge management research: State of the art and research opportunities". International Journal of Production Economics, 182, pp. $276-292$.

[6] Ørnulf Jan Rødseth, 2018. "Assessing business cases for autonomous and unmanned ships". In Proceedings of NAV 2018.

[7] Beamon, B. M., 1998. "Supply chain design and analysis:: Models and methods". International Journal of Production Economics, 55(3), pp. $281-294$.

[8] Kretschmann, L., Burmeister, H.-C., and Jahn, C., 2017. "Analyzing the economic benefit of unmanned autonomous ships: An exploratory cost-comparison between an autonomous and a conventional bulk carrier". Research in Transportation Business Management, 25, pp. $76-86$. New developments in the Global Transport of Commodity Products. 
[9] Rødseth, J., and Burmeister, H.-C., 2015. MUNIN 10.2 New ship designs for autonomous vessels, oct.

[10] SAE, 2018. "Taxonomy and definitions for terms related to driving automation systems for on-road motor vehicles j3016_201806". p. 35 .

[11] Ørnulf Jan Rødseth, Nordahl, H., and Åse Hoem, 2018. "Characterization of autonomy in merchant ships". In 2018 OCEANS MTS/IEEE Kobe Techno-Oceans (OTO), pp. 1-7.

[12] ISO, 2019. Ships and marine technology - terminology related to automation of maritime autonomous surface ships (MASS) internal committee working draft 1 , aug.

[13] Grøtli, E. I., Reinen, T. A., Grythe, K., Transeth, A. A., Vagia, M., Bjerkeng, M., Rundtop, P., Svendsen, E., Rødseth, J., and Eidnes, G., 2015. "Seatonomy". In OCEANS 2015 - MTS/IEEE Washington, pp. 1-7.

[14] Grøtli, E. I., Bjerkeng, M., Rundtop, P., Vagia, M., Haugli, F. B., and Transeth, A. A., 2017. "Canvas as a design tool for autonomous operations: With application to net inspection of a sea based fish farm using an underwater vehicle". In OCEANS 2017 Aberdeen, pp. 1-5.
[15] Bolbot, V., Theotokatos, G., Bujorianu, L. M., Boulougouris, E., and Vassalos, D., 2019. "Vulnerabilities and safety assurance methods in cyber-physical systems: A comprehensive review". Reliability Engineering System Safety, 182, pp. 179 - 193.

[16] Ørnulf Jan Rødseth, and Burmeister, H.-C., 2015. "Risk assessment for an unmanned merchant ship". TransNav, the International Journal on Marine Navigation and Safety of Sea Transportation, 9(3), pp. 357-364.

[17] Banda, O. A. V., Kannos, S., Goerlandt, F., van Gelder, P. H., Bergström, M., and Kujala, P., 2019. "A systemic hazard analysis and management process for the concept design phase of an autonomous vessel”. Reliability Engineering System Safety, 191, p. 106584.

[18] USA Department of Defence, 2000. Department of defence standard practice system safety, may.

[19] Wennersberg, L. A. L., Nordahl, H., Bolbot, V., and Theotokatos, G., 2019. AUTOSHIP D2.1 Complete supply chain mapping and identifications of interactions between SSS and IWW demonstrators, nov. 